\title{
EVALUATION OF ORTHOSTATIC HYPOTENSION BY USING HUTT
}

\author{
Qurban Hussain Khan, Muhammad Asad, Waqas Baloch, Mian Muhammad Amer Naseem, Muhammad Shabbir, \\ Azmat Hayat \\ Armed Forces Institute of Cardiology/National Institute of Heart Disease (AFIC/NIHD)/National University of Medical Sciences (NUMS) \\ Rawalpindi Pakistan
}

\begin{abstract}
Objective: To determine the prevalence of orthostatic hypotension in our patient population by using Head Up Tilt Test (HUTT).

Study Design: Descriptive cross-sectional study.

Place and Duration of Study: Armed Forces Institute of Cardiology \& National institute of heart diseases, from Jan 2019 to Jan 2020.

Methodology: Head Up Tilt Test registry was used as a data collection tool which consisted of gender, age, symptoms, number of episodes, results, baseline blood pressure systolic, baseline blood pressure diastolic, mean blood pressure, baseline heart rate. All patients from age 20-80 undergoing Head Up Tilt Test examination were included while patients already diagnosed with orthostatic hypotension, vasovagal syncope, and cardiac myopathy were excluded from the study.

Results: Total 1587 individuals were enrolled in study, I1216 (76.6\%) were males and 371 (23.4\%) were females. The mean age of the study population was $45.63 \pm 19$ years. The results determined $67.6(4.2 \%)$ were negative, $7(0.4 \%)$ had Vasodepressive Response of vasovagal reflex, $1435(90.4 \%)$ had positive type 1; while $1(0.1 \%)$ had positive type 2; and $4(0.3 \%)$ had positive type 3; $62(3.9 \%)$ had mixed vasovagal reflex, $1(0.1 \%)$ had late orthostatic response and $10(0.6 \%)$ had orthostatic hypotension.

Conclusion: Tilt-table testing is an effective method to diagnose orthostatic hypotension.
\end{abstract}

Keywords: HUTT, Orthostatic hypotension, Syncope, Vasovagal reflex.

This is an Open Access article distributed under the terms of the Creative Commons Attribution License (http://creativecommons.org/licenses/by/4.0), which permits unrestricted use, distribution, and reproduction in any medium, provided the original work is properly cited.

\section{INTRODUCTION}

Orthostatic hypotension $(\mathrm{OH})$ is defined as a reduction in systolic blood pressure $(\mathrm{BP})$ of at least $20 \mathrm{~mm} \mathrm{Hg}$ or $10 \mathrm{~mm} \mathrm{Hg}$ reduction in diastolic BP upon standing or during a head up tilt test to at least $60^{\circ}$. $\mathrm{OH}$ is highly prevalent in the community, but due to diverse clinical presentations, it is often unrecognized until late in the clinical course ${ }^{2}$. It is associated with significant morbidity and loss of autonomy in the elderly. $\mathrm{OH}$ is associated with an increased risk of falls, heart failure, renal failure, stroke, atrial fibrillation, hospitalization, and death ${ }^{2}$.

The 3 common variants are: Classical $\mathrm{OH}$, delayed $\mathrm{OH}$, and initial $\mathrm{OH}^{3}$. Classical $\mathrm{OH}$ is characterised by a sustained reduction of at least $20 \mathrm{~mm} \mathrm{Hg}$ of systolic BP or $10 \mathrm{~mm} \mathrm{Hg}$ of diastolic

Correspondence: Dr Qurban Hussain Khan, EP Department, AFIC/ NIHD Rawalpindi Pakistan
BP within 3 minutes of standing or tilt-table testing. In delayed $\mathrm{OH}$ there is a sustained reduction in BP which occurs after 3 minutes of standing or upright tilt. Initial $\mathrm{OH}$ is defined by a transient reduction in $\mathrm{BP}$ (defined as a reduction $\geq 40 \mathrm{~mm}$ $\mathrm{Hg}$ systolic $\mathrm{BP}$ and/or $\geq 20 \mathrm{~mm} \mathrm{Hg}$ diastolic $\mathrm{BP}$ ) within 15 seconds of standing 3 .

Both vasovagal syncope and delayed $\mathrm{OH}$, though different disorders, cause blood pressure falls and syncope. The key distinguishing feature is rate of blood pressure fall. In vasovagal syncope, tilt table testing shows a sudden fall in blood pressure, usually with bradycardia and prodromal symptoms. This blood pressure fall may occur several minutes after upright tilting of the table, whereas in neurogenic $\mathrm{OH}$ the blood pressure falls almost immediately upon upright tilting. Delayed $\mathrm{OH}$ is typically associated with a slow gradual reduction in blood pressure, analogous to a ball rolling down a slope. In addition 
in vasovagal syncope, there is a fall in heart rate which accompanies the BP fall, whereas in delayed $\mathrm{OH}$, there is typically no decrease in heart rate ${ }^{4}$.

Tilt table testing may demonstrate the reproduction of vasovagal syncope in a controlled, laboratory setting. Positive responses in patients with neurally mediated syncope are $61-69 \%$ and specificity is high (92-94\%) ${ }^{5}$. The most commonly used protocol includes initially tilting the table to $70^{\circ}$, a passive non-medicated phase of $20 \mathrm{minu}-$ tes, administration of 300-400 micrograms of sublingual nitroglycerine after the 20th minute, followed by an additional 20 minutes of standing 5 .

The main indication for tilt testing is the confirmation of a diagnosis of vasovagal syncope in patients in whom it has been suspected from the initial history. This typically includes cases involving unexplained syncope in a high-risk setting or multiple recurrent episodes when a cardiovascular cause has been excluded. Tilt testing is also performed to demonstrate susceptibility to vasovagal syncope ${ }^{5}$.

Other tilt-table testing indications include distinguishing between reflex syncope and orthostatic hypotension or falls; and also transient loss of consciousness due to epilepsy or psychiatric problems ${ }^{5}$.

\section{METHODOLOGY}

A descriptive cross-sectional study was conducted at Electrophysiology department of Armed Forces Institute of Cardiology \& National institute of heart diseases for a period of one year from January 2019 to January 2020. Prior to data collection approval from the Institutional Ethical Review Board was taken. Head up Tilt Test registry was used as a data collection tool which consisted of gender, age, symptoms, number of episodes, results, baseline BP systolic, baseline $\mathrm{BP}$ diastolic, mean BP, baseline HR. Total 1587 individuals were enrolled in the study through non-probability consecutive sampling technique after satisfying the inclusion criteria which was all patients from age 20 to 80 undergoing HUTT examination while excluding patients already diagnosed with orthostatic hypotension, patients already diagnosed with vasovagal syncope. Patients suffering from any cardiac myopathy were excluded from the study. Descriptive statistics was run to calculate Frequency/percentage, Mean \pm SD as variables are quantitative.

\section{RESULTS}

Total 1587 individuals were enrolled in study. One thousand two hundred and sixteen (76.6\%) were Males and 371 (23.4\%) were Females. The mean age of the study population was $45.63 \pm 19$. The results of the tilt test depicted 67.6

Table-I: Demographic characteristics of the study population.

\begin{tabular}{|c|c|}
\hline Variables & Mean \pm SD $/ n(\%)$ \\
\hline \multicolumn{2}{|l|}{ Gender } \\
\hline Males & $1216(76.6 \%)$ \\
\hline Females & $371(23.4 \%)$ \\
\hline Age & $45.63 \pm 19.67$ \\
\hline \multicolumn{2}{|l|}{ Symptoms } \\
\hline Syncope & $919(57.9 \%)$ \\
\hline Fitness \& categorization & $11(0.7 \%)$ \\
\hline Determine Arrythmia & $24(1.5 \%)$ \\
\hline Conduction Abnormalities & $45(2.8 \%)$ \\
\hline Find any Pauses & $1(0.1 \%)$ \\
\hline $\begin{array}{l}\text { Weakness while prolong } \\
\text { standing }\end{array}$ & $11(0.7 \%)$ \\
\hline Sinus Tachy & $1(0.1 \%)$ \\
\hline Blackout & $35(2.2 \%)$ \\
\hline Dizziness & $247(15.6 \%)$ \\
\hline Palpitation & $16(1 \%)$ \\
\hline VPC & $1(0.1 \%)$ \\
\hline Dizziness \& Blackout & $276(17.4 \%)$ \\
\hline \multicolumn{2}{|l|}{ No. of Episodes } \\
\hline 1 Time & $542(34.2 \%)$ \\
\hline 2-5 times & $501(31.6 \%)$ \\
\hline 6-10 times & $37(2.3 \%)$ \\
\hline Many times & $157(9.9 \%)$ \\
\hline OFF and ON & $182(11.5 \%)$ \\
\hline Often & $85(5.45 \%)$ \\
\hline Sometimes & $1(0.1 \%)$ \\
\hline
\end{tabular}

(4.2\%) were negative, 7 (0.4\%) had Vasodepressive Response of vasovagal reflex, 1435 (90.4\%) had positive type 1; a mixed vasovagal response while $1(0.1 \%)$ had positive type 2; cardioinhibitory vasovagal response and $4(0.3 \%)$ had positive type 3; vasodepressor vasovagal response, $62(3.9 \%)$ have mixed vasovagal reflex, $1(0.1 \%)$ 
had late orthostatic response and $10(0.6 \%)$ had orthostatic hypotension. The base line BP systolic was $136.83 \pm 16.951$, the base line BP diastolic was $81.99 \pm 20.99$. The Mean BP recorded was $95.98 \pm$ 14.924 and the baseline HR was $71.65 \pm 14.084$.

Table-II: Results of Head Up Tilt Test.

\begin{tabular}{l|c}
\hline & $\mathbf{n ~ ( \% )}$ \\
\hline Results & $67(4.2 \%)$ \\
Negative & $7(0.4 \%)$ \\
\hline Vasodepressive response of vasovagal reflex \\
\hline Positive Type 1 & $1435(90.4 \%)$ \\
Positive Type 2 & $1(0.1 \%)$ \\
Positive Type 3 & $4(0.3 \%)$ \\
\hline Mixed vasovagal reflex & $62(3.9 \%)$ \\
Late orthostatic response & $1(0.1 \%)$ \\
Orthostatic hypotension & $10(0.6 \%)$ \\
\hline Baseline BP systolic & $136.83 \pm 16.951$ \\
\hline Baseline BP Diastolic & $81.99 \pm 20.99$ \\
\hline BP Mean & $95.98 \pm 14.924$ \\
\hline HR Baseline & $71.65 \pm 14.084$ \\
\hline
\end{tabular}

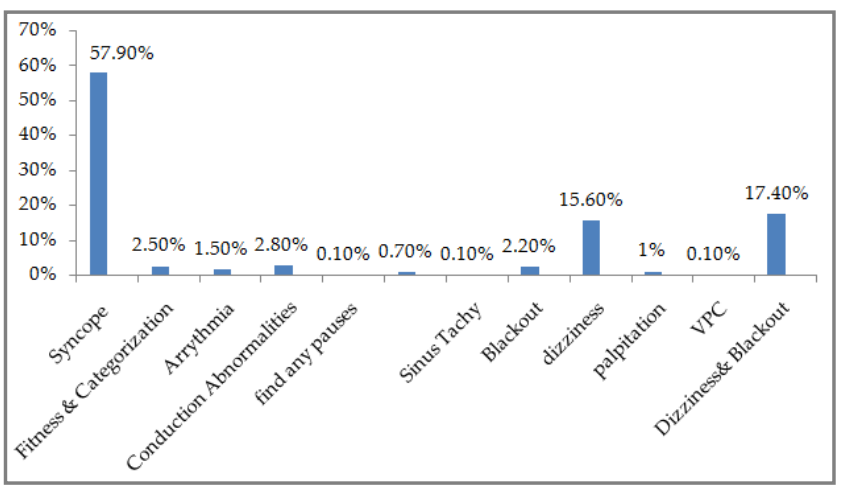

Figure: Symptoms of the study population.

\section{DISCUSSION}

The data revealed that an overwhelming majority of the patients who underwent tilt-table testing revealed a positive response for vasovagal syncope $(95.1 \%)$. Only $0.6 \%$ of the patients were diagnosed with orthostatic hypotension. At initial perusal this may seem surprising given the suspected prevalence of $\mathrm{OH}$ in the communitythe ARIC (Atherosclerosis Risk In Communities) study, a community-based prospective cohort of 15,792 middle-aged adults 45-64 years of age, showed that $\mathrm{OH}$ was present in $5 \%$ of individuals ${ }^{7-11}$.
One possible explanation may be that in the Armed Forces Institute of Cardiology, where the study was carried out, many soldiers specifically with clinical presentations highly typical of vasovagal syncope are referred for tilt-table testing. This may have skewed the positive results in favour of vasovagal syncope. The population for this study had a mean age of 45, significantly lower than the ARIC study, which can also be accounted for by the number of soldiers that underwent tilt testing. $\mathrm{OH}$ is less prevalent in younger populations ${ }^{12-18}$, and this may explain the lower than expected frequency of $\mathrm{OH}$ diagnosis in the study population.

Another possible explanation for the low prevalence of $\mathrm{OH}$ in the study population may be that very few patients with $\mathrm{OH}$ are being referred for tilt-testing because they are being diagnosed using other clinical tests such as the Active Standing Test ${ }^{2}$. Once diagnosed in this manner there is no further diagnostic benefit accrued by undergoing a tilt-table test, which has been showed to be less sensitive than the Active Standing Test ${ }^{2}$.

It is also possible that $\mathrm{OH}$ is being underdetected using tilt-table testing ${ }^{19-21}$. There is considerable overlap between the clinical presentations in vasovagal syncope and $\mathrm{OH}$, and syncope may be a feature of either. The pattern of fall in BP and change in heart rate is one way of discriminating between the two conditions but sometimes even then it may be difficult to give a definitive diagnosis ${ }^{4}$.

Once diagnosed with $\mathrm{OH}$, treatment is geared towards relief of symptoms rather than correcting orthostatic hypotension. Asymptomatic $\mathrm{OH}$ is common and of uncertain clinical relevance. Symptomatic $\mathrm{OH}$ management addresses modifiable contributing factors (anemia, drugs, dehydration), and employs initially non-pharmacologic strategies, and, if required, pharmacologic therapy. Orthostatic stresses vary with circumstances during the day, and so it is essential to have a patient-oriented approach emphasizing education and non-pharmacologic strategies to minimize orthostatic stress $6,18-21$. 


\section{CONCLUSION}

Tilt-table testing is an effective method to diagnose orthostatic hypotension.

\section{CONFLICT OF INTEREST}

This study has no conflict of interest to be declared by any author.

\section{REFERENCES}

1. Frith J. Diagnosing orthostatic hypotension: a narrative review of the evidence. British Medical Bulletin 2015; 115(1): 123-34.

2. Aydin A, Soysal P, Isik A. Which is preferable for orthostatic hypotension diagnosis in older adults: active standing test or head-up tilt table test?. Clinical Interventions in Aging, 2017; 12(1): 207-12.

3. Shaw BH, Borrel D, Sabbaghan K, Kum C, Yang Y, Robinovitch $\mathrm{SN}$, et al. Relationships between orthostatic hypotension, frailty, falling and mortality in elderly care home residents. BMC geriatrics 2019; 19(1): 80-85.

4. Freeman R, Abuzinadah A, Gibbons C, Jones P, Miglis M, Sinn D. Orthostatic Hypotension. J Ameri College Cardiology 2018; 72(11): 1294-09.

5. Gibbons $\mathrm{CH}$, Schmidt P, Biaggioni I, Frazier-Mills C, Freeman R, Isaacson $\mathrm{S}$, et al. The recommendations of a consensus panel for the screening, diagnosis, and treatment of neurogenic orthostatic hypotension and associated supine hypertension. J Neurology 2017; 264(8): 1567-82.

6. Shaw BH, Garland EM, Black BK, Paranjape SY, Shibao CA, Okamoto LE, et al. Optimal diagnostic thresholds for diagnosis of orthostatic hypotension with a "sit-to-stand test". J Hypertension 2017; 35(5): 1019.

7. Palma JA, Kaufmann H. Epidemiology, diagnosis, and management of neurogenic orthostatic hypotension. Movement disorders clinical practice 2017; 4(3): 298-08.

8. Jones P, Shaw B, Raj S. Orthostatic hypotension: managing a difficult problem. Expert Review of Cardiovascular Therapy
2015; 13(11): 1263-76.

9. Fedorowski A, van Wijnen VK, Wieling W. Delayed orthostatic hypotension and vasovagal syncope: a diagnostic dilemma. Clinical Autonomic Res 2017; 27(4): 289-91.

10. Stewart JM, Shaban MA, Fialkoff T, Tuma Marcella B, Visintainer $\mathrm{P}$, Terilli $\mathrm{C}$, et al. Mechanisms of tilt induced vasovagal syncope in healthy volunteers and postural tachycardia syndrome patients without past history of syncope. Physiological reports2017; 7(13): e14148.

11. Medow MS, Merchant S, Suggs M, Terilli C, O'Donnell-Smith B, Stewart JM. Postural heart rate changes in young patients with vasovagal syncope. Pediatrics 2017; 139(4): p.e20163189.

12. Simova, I, Role of tilt-table testing in syncope diagnosis and management. E-J Cardiol Prac 2015; 13(1): 1-5.

13. Kohno R, Adkisson WO, Benditt DG. Tilt table testing for syncope and collapse. Herzschrittmachertherapie \pm Elektrophysiologie 2018; 29(2): 187-92.

14. Furukawa T. Role of head-up tilt table testing in patients with syncope or transient loss of consciousness. J arrhythmia 2017; 33(6): 568-71.

15. Teodorovich N, Swissa M. Tilt table test today-state of the art. World J cardiology 2016; 8(3): p.277.

16. Zyśko D, Fedorowski A, Nilsson D, Rudnicki J, Gajek J, Melander $\mathrm{O}$, et al. Tilt testing results are influenced by tilt protocol. Ep Europace 2016; 18(7): 1108-12.

17. Çakır C, Ceylan Y, Hakgor A. Reproducibility of Tilt-table Test with Different Dosages of Sublingual Nitroglycerin. Eastern J Med 2016; 24(3): 350-54.

18. Casiglia E, Jordan J. Orthostatic hypotension: new views for an old problem. J Hyper 2017; 35(5): 947-49.

19. Arnold AC, Raj SR. Orthostatic hypotension: a practical approach to investigation and management. Can J Cardiol 2017; 33(12): 1725-28

20. Rukmani MR, Yadav R, Pal PK, Sathyaprabha TN. Clinical management of neurogenic orthostatic hypotension. Annals Movement Disorders 2019; 2(3): p.91-95.

21. Jones J, Kuritzky L. Recognition and management of orthostatic hypotension in primary care. J Family Practice 2018; 67(8 Suppl): S25-S30. 